\title{
Dark Matter from Early Decays
}

\author{
Manoj Kaplinghat \\ Department of Physics and Astronomy, 4129 Frederick Reines Hall \\ University of California, Irvine, California 92697, USA
}

(Dated: July 28, 2021)

\begin{abstract}
Two leading dark matter candidates from supersymmetry and other theories of physics beyond the standard model are WIMPs and weak scale gravitinos. If the lightest stable particle is a gravitino, then a WIMP will decay into it with a natural lifetime of order a month $\sim M_{\mathrm{pl}}^{2} / M_{\text {weak }}^{3}$. We show that if the bulk of dark matter today came from decays of neutral particles with lifetimes of order a year or smaller, then it could lead to a reduction in the amount of small scale substructure, less concentrated halos and constant density cores in the smallest mass halos. Such beneficial effects may therefore be realized naturally, as discussed by Cembranos, Feng, Rajaraman, and Takayama, in the case of supersymmetry.
\end{abstract}

\section{INTRODUCTION}

We have many independent lines of observational evidence that point to the existence of dark matter in the universe. The dark matter particle, however, has not been observed. Most theories of physics beyond the standard model that have new physics at the weak scale, naturally predict the existence of WIMP (Weakly Interacting Massive Particle) dark matter. The weak interaction cross-section endows the WIMP with the right relic density to make up a substantial fraction of the energy density of the universe. Thus most cosmological studies of dark matter assume that it is a stable cold particle with interaction cross-sections comparable to the weak interaction cross-sections.

Within theories of physics beyond the standard model, one also has the gravitino (or other gravitationally coupled particles). Recent work by Feng, Rajaraman and Takayama 1] provided an interesting twist to the old dark matter tale. They observed that if the gravitino is the lightest supersymmteric particle (LSP), then the next lightest particle (NLSP) can decay into the gravitino with a lifetime that is long since the gravitino couples only gravitationally. The natural time scale for this decay is $M_{\mathrm{pl}}^{2} / M_{\text {weak }}^{3} \sim$ month. This opens up the possibility that at least a fraction of the dark matter we observe today comes from decays when the universe was days to years old. We note that similar models may be constructed in the context of cosmologies with extra dimensions 2]. Two early universe constraints on these models are that the electromagnetic decay products must not distort the black body spectrum appreciably and the light element abundances from BBN must match with observations. Recent work has shown that there are regions of parameter space where all these conditions may be met [3, 4, 5, 6, 7]. In the present work we work out the late time consequences of these decays.

Another way to get dark matter to decay around similar epochs is to kinematically suppress the decays such that the lifetime is orders of magnitude larger than the "natural" time scale. Profumo et al. 8] provided the first example of such a decay that arises in supersymmetric theories. The consider the decay of stau into the LSP with a mass splitting smaller than the tau lepton mass. In this case, the two body decay is forbidden and the stau can only decay into 4 daughter particles. The 4-body decay of a charged slepton is suppressed by the NLSP-LSP mass splitting as $(M / \Delta M)^{8}$. Thus with $\Delta M / M \sim 10^{-3}$, the stau lifetime is of order a few weeks 8].

Sigurdson and Kamionkowski [9] and then Profumo et al. 8] worked out the impact of the charged stau decay on the matter and CMB power spectrum. They found that the dark matter power spectrum was suppressed due to the coupling of the charged NLSP to the photon-baryon system. This coupling damps the matter power spectrum once a perturbation mode enters the horizon.

In this paper we point out that the large suppression of the matter power spectrum is possible even if the NLSP is not charged. Unless the mass splitting between the NLSP and LSP is fine-tuned to be small, we point out that the decay will impart a large velocity to the LSP. Effectively the LSP will act like warm dark matter. This leads to a cut-off in the matter power spectrum. We calculate shape of the power spectrum given the lifetime and the masses of the particles involved.

We also calculate the effect of the smaller phase space density that results from this decay on dark matter halos. They could (for small enough primordial phase space density) be instrumental in shaping the smallest mass halos, lowering the fraction of mass in sub-halos, and reducing the concentration of larger mass halos. Stringent constraints on these decays come from the requirement that the universe be reionized by a redshift of six or higher. If the present hints 10 for early reionization are borne out by future data, then this could rule out a big chunk of the supersymmetric parameter space in which the gravitino is the lightest particle.

Cembranos et al. [11] show that the beneficial effects mentioned above may be obtained in a large region of the super-symmetric parameter space. The prospects for testing this region of parameter space wherein we have early decays is promising. These models will be tested by observations made with NGST of the high redshift universe (e.g., [12]), by future surveys of strong lens systems (e.g., [13]), weak lensing measurements (e.g., [14]), as well as the more traditional $\mathrm{CMB}$ and large scale struc- 
ture observations.

The dark matter observed today could also be a mixture of particles produced during the reheating phase and those arising from the decays. For the gravitino example, the two contributions are expected to be comparable for a reheating temperature of $10^{10} \mathrm{GeV}$. This kind of mixed model would ameliorate the rapid drop in power on small scales.

Dark matter from decays have a non-thermal momentum distribution. Lin et al. 15], Hisano et al. 16], Kitano and Low 17] studied different models of nonthermal WIMP dark matter and their effect on the small scale structure. The generic cosmological effects they found are similar to what we discuss here because the effects stem from the large velocity dispersion. Decays leading to a large velocity dispersion were also considered briefly by Hogan and Dalcanton [18] in their work on the astrophysical consequences of a small primordial phase space density.

\section{DARK MATTER FROM DECAYS}

Consider the decay process, DDM $\rightarrow \mathrm{DM}+\mathrm{L}$, where $\mathrm{L}$ denotes "light" particles with mass $m_{1}, \mathrm{DM}$ is the dark matter today with mass $m$ and DDM is the parent particle with mass $M$. When we derive the perturbations, we will be enforce the limit $m_{1}<<m$ as is appropriate for supersymmetric models (e.g., [4]). We will also assume that DDM and DM are neutral, for example, a sneutrino decaying into a neutrino and a gravitino. All of the effects we mention here are relevant also for charged particle decay. In addition, the coupling of the charged parent particle to the photon-baryon system results in the damping of perturbations that come into the horizon when the charged parent particles dominate the non-relativistic matter density [9]. We will work out the physics and astrophysics of charged particle decay in a future publication.

For a generic n-body decay, neglecting Pauli-blocking factors and inverse decays, the change in the phase space due to decays is given by

$$
\dot{f}_{\mathrm{DDM}}\left(p_{\mathrm{DDM}}\right)=-\frac{a M}{E_{\mathrm{DDM}} \tau} f_{\mathrm{DDM}}\left(p_{\mathrm{DDM}}\right),
$$

where $E_{\mathrm{DDM}}^{2}=p_{\mathrm{DDM}}^{2}+M^{2}$ and $f_{\mathrm{DDM}}$ is the phase space distribution of the DDM particles. The over-dot denotes derivative with respect to the conformal time, $d \eta=d t / a$ and $a$ is the scale factor. Specializing to two-body decays, one can show that the DM phase space is populated by the DDM decays according to the equation [19, 20]:

$$
\dot{f}_{\mathrm{DM}}\left(p_{\mathrm{DM}}\right)=\frac{a M^{2}}{2 \tau E_{\mathrm{DM}} p_{\mathrm{DM}} p_{\mathrm{C} M}} \int_{E_{1}}^{E_{2}} d E f_{\mathrm{DDM}}(p),
$$

where $E_{2,1}=\left(0.5 E_{\mathrm{DM}} m_{0}^{2} \pm p_{\mathrm{DM}} p_{\mathrm{C} M} M\right) / m_{\mathrm{DM}}^{2} \cdot p_{\mathrm{C} M}$ is the center-of-mass momentum and $m_{0}^{2} \equiv M^{2}+m_{\mathrm{DM}}^{2}-$ $m_{\mathrm{L}}^{2}$. An analogous equation holds for the other daughter particle. Note that for a two-body decay we have lifetime $\tau=8 \pi M^{2} / p_{\mathrm{CM}}|\mathcal{M}|^{2}$ where $|\mathcal{M}|^{2}$ is the quantum mechanical amplitude for the decay process. Also note that since the decay happens when the DDM particle is cold, $p_{\mathrm{C} M}$ is an accurate measure of the momentum imparted to the daughter particles.

The equations for the change in the phase space distribution due to the decays, Eq. 1 and Eq. 2 can be integrated to yield the following equations for the change in the density of DM and DDM.

$$
\begin{aligned}
\dot{\rho}_{\mathrm{DDM}}+3 \frac{\dot{a}}{a} \rho_{\mathrm{DDM}} & =-\frac{a}{\tau} \rho_{\mathrm{DDM}}, \\
\dot{\rho}_{\mathrm{DM}}+3 \frac{\dot{a}}{a}\left(\rho_{\mathrm{DM}}+P_{\mathrm{DM}}\right) & =\frac{a m_{0}^{2}}{2 \tau M^{2}} \rho_{\mathrm{DDM}},
\end{aligned}
$$

where $P_{\mathrm{DM}}$ is the pressure of $\mathrm{DM}$ and it is comparable to $\rho_{\mathrm{DM}}$ at early times when the bulk of decay occurs. An equation for "L" particles may be written down by inspection of the DM equation.

We will also need to calculate the phase space distribution of DM particles. One can show that this is (in the limit of completely non-relativistic decay) given by

$$
f_{\mathrm{DM}}(q, a)=\frac{(2 \pi)^{2} \Omega_{M} \rho_{\mathrm{crit}} t_{q}}{m q^{3} \tau} \exp \left(-\frac{t_{q}}{\tau}\right) \Theta\left(a p_{\mathrm{C} M}-q\right),
$$

where $q$ is the comoving momentum of the DM particle and $t_{q}=t\left(a=q / p_{\mathrm{C} M}\right)$. We have assumed that the decays are happening during radiation domination and hence $t_{q} \propto q^{2}$ and $f_{\mathrm{DM}}(q) \propto q^{-1} \exp \left(-q^{2} /\left[2 p_{\mathrm{C} M}^{2} \tau \dot{a}\right]\right)$.

\section{PERTURBATIONS}

We now write down the equations for the perturbations in DDM, DM and L particles. The first result is that the perturbation in density relative to the mean for the DDM particles is unchanged. This is derived by using the fact that the DDM particles are non-relativistic. The physical content of this statement is that since the DDM particles do not have a large peculiar velocity during decay, the only effect of the decay is to remove the same fraction of the DDM particles from every region of space.

To calculate the perturbations explicitly, we write it as a sum of two terms:

$$
\delta f_{\mathrm{DM}, \ell}(k, q, a)=\delta f_{\mathrm{DM}, \ell}^{(a)}(k, q, a)+\delta f_{\mathrm{DM}, \ell}^{(b)}(k, q, a) .
$$

The first term $\delta f_{\mathrm{DM}, \ell}^{(a)}(k, q, a)$ is chosen to satisfy the collisionless Boltzmann equation for a massive particle and may be calculated in a manner analogous to the perturbations in the massive neutrino phase space [21]. The second term is harder to calculate numerically. One can make approximations similar to the one made in the previous section and obtain:

$$
\delta f_{\mathrm{DM}, \ell}^{(b)}(k, q, a)=f_{\mathrm{DM}}(q, a) h\left(k, a_{q}\right) \jmath_{\ell}\left(k \omega_{q}\left(a, a_{q}\right)\right),
$$


where $a_{q}=q / p_{\mathrm{C} M}, \omega_{q}(x, y)=\int_{y}^{x} d a(q / \epsilon(q, a)) / \dot{a}$ and $\epsilon(q, a)=\sqrt{m^{2} a^{2}+q^{2}}$ is the comoving energy. Note that $\omega_{q}(x, y)$ is the comoving distance traversed by a particle with comoving momentum $q$ between $x$ and $y$. $h(k, a)$ is the perturbation to the trace of the the synchronous gauge spatial metric in fourier space written as $\delta_{i j} \delta(\mathbf{k}) / 3+\delta_{i j} h(k, a)+\left(\hat{k}_{i} \hat{k}_{j}-\delta_{i j} / 3\right) \alpha(k, a)[21]$.

We have modified CMBfast 22] to incorporate the above physics. The physics of how the decay affects the dark matter perturbations is simple. The decay of DDM to DM provides a large momentum to the DM particle. This implies that the mean free path of DM is larger than in the standard case. Perturbations on scales smaller than the "mean free path" of the DM particle cannot survive.

How is this manifested in the Boltzmann equation? The solution of the linearized collisionless Boltzmann equation (valid when the decay term can be neglected) can be written as

$$
\begin{aligned}
\delta \rho\left(a_{0}\right) & =(2 \pi)^{-2} a_{0}^{-4} \int_{0}^{a_{0}} d a \int_{0}^{\infty} d q \epsilon q^{3} \frac{d f_{0}}{d q}[ \\
& \left.-\frac{h^{\prime}}{3} j_{0}\left(k \omega_{q}(a, 0)\right)+\frac{2}{3} \alpha^{\prime} j_{2}\left(k \omega_{q}(a, 0)\right)\right],
\end{aligned}
$$

where $h^{\prime}$ and $\alpha^{\prime}$ denote derivatives with respect to scale factor $a$. The free-streaming effect comes in through the effect of the function $\omega_{q}$. We note that the $j_{0}$ and $j_{2}$ terms result from the plane wave expansion of $\exp \left(\imath k \omega_{q}(a, 0)\right)$. In the limit of $m \rightarrow 0$, we have a plane wave (which is how the photons free-stream after decoupling). In the other limit of $m \rightarrow \infty, j_{0} \rightarrow 1$ and $j_{2} \rightarrow 0$, and we obtain the evolution of the CDM density perturbations. Similar expressions were first derived by Bond and Efstathiou [23], and Brandenberger, Kaiser and Turok 24]. On small scales, one may make further approximations and write $\delta \rho\left(a_{0}\right)=(2 \pi)^{-2} a_{0}^{-4} \int_{0}^{a_{0}} d a\left(h^{\prime}+\right.$ $\left.\alpha^{\prime}\right) / 3 \int d q q^{2} \epsilon f_{0} d \ln \left(w_{q}\right) / d \ln (q) \cos \left(k \omega_{q}\right)$. Note that when $k \omega_{q}\left(a, q / p_{\mathrm{C} M}\right)$ gets large, we get no contributions to the integral due to cancellations from rapid oscillations. The power spectrum is thus suppressed.

A point of interest in this discussion is that the quantity that determines the damping of the power spectrum on small scales is not the canonically defined mean free path $\int_{\tau}^{t_{\text {eq }}} d t v(t) / a(t)$. Damping occurs when $k \omega_{q}\left(a, q / p_{\mathrm{C} M}\right)>1$; very roughly, this condition picks out scales smaller than $0.005\left(Q /\left[M_{\odot} / \mathrm{pc}^{3} /\left(\mathrm{km} \mathrm{s}^{-1}\right)^{3}\right]\right)^{-1 / 3} \mathrm{Mpc}$.

What are the natural variables to describe this class of "warm dark matter" models? We may take $a_{\mathrm{dec}}$ and $m / p_{\mathrm{C} M}$ to be the two variables. $a_{\mathrm{dec}}$ is the scale factor of the universe when the age is equal to the lifetime $\tau$. The cosmological consequences depend only on these two variables.

If we specialize to the case of decays in the radiation dominated era, then we will find that there is just one variable that adequately describes the cosmological effects. For those decays that release EM energy, decays must occur in the radiation dominated era in order to not distort the CMB black body spectrum beyond the $10^{-4}$ level.

A physically motivated variable is phase space density of DM particles in the early universe. We will adopt the definition of Hogan and Dalcanton [18] who defined $Q=$ $\rho /\left\langle v^{2}\right\rangle$. For times much larger than the decay lifetime, we have the exact relation

$$
Q=10^{-24}\left(\frac{m}{p_{\mathrm{C} M} a_{\mathrm{dec}}}\right)^{3} \frac{M_{\odot} / \mathrm{pc}^{3}}{\left(\mathrm{~km} \mathrm{~s}^{-1}\right)^{3}} .
$$

We will see that as a first approximation, the power spectrum only depends on $Q$. For reference, we note that in the case of the slepton decaying to the gravitino, $Q=$ $2.1 \times 10^{-3}\left(2 p_{\mathrm{C} M} / M\right)^{3}(M / \mathrm{TeV})^{4.5} M_{\odot} / \mathrm{pc}^{3} /\left(\mathrm{km} \mathrm{s}^{-1}\right)^{3}$.

The net result on the power spectrum can be written in a manner analogous to the massive neutrino case. The power-spectrum is suppressed on scales smaller than $\lambda_{c}$. Here we provide a simple fitting formula that encapsulates the basic features of the power spectrum at the $25 \%$ level for lifetimes less than about a year and $k<25 h /$ Mpc.

$$
\begin{aligned}
P_{\mathrm{DM}}(k) & =P_{\mathrm{CDM}}\left(\frac{1}{2} \exp \left(-\left(k \lambda_{c}\right)^{2} / 2\right)\right. \\
& \left.+\frac{1}{2}\left[1+\left(k \lambda_{c}\right)^{3}\right]^{-1}\right)^{2},
\end{aligned}
$$

where $\lambda_{c}=0.0198 / Q^{0.275} \mathrm{Mpc}$. At this level of accuracy, we thus have a one parameter family of models.

The above power spectrum should be compared to the power spectrum in Warm Dark Matter (WDM) models. Bode, Ostriker and Turok 25] quote $P_{\mathrm{WDM}}=$ $P_{\mathrm{CDM}}\left(1+(\alpha k)^{2 \nu}\right)^{-10 / \nu}$ based their analytic work and the exact results of $\mathrm{Ma}$ [26], where $\nu=1.2$ and $\alpha=0.048 h^{-1} \operatorname{Mpc}\left(\Omega_{\mathrm{WDM}} h^{2} / 0.169\right)^{0.15}(\mathrm{keV} / m)^{1.15}$ for a fermionic WDM with two internal degrees of freedom. Note that the asymptotic power-law in the two models are different; the decay model has comparatively more power due to its non-thermal distribution. Looking at the variance $\sigma(M)$, we find that the modified DM power spectrum with $\lambda_{c}=0.1 \mathrm{Mpc} / h$ is a good fit to the variance function for a $1 \mathrm{keV}$ WDM model. Bode, Ostriker and Turok 25] find that the large scale structure constraints are met by a WDM model with $1 \mathrm{keV}$ particle. Thus, $\lambda_{c} \lesssim 0.1 \mathrm{Mpc} / h$ will also reproduce the large scale structure we observe. For reference we observe that a keV WDM implies $Q=5 \times 10^{-4} M_{\odot} / \mathrm{pc}^{3} /\left(\mathrm{km} \mathrm{s}^{-1}\right)^{3}$. From the approximate formula following Equation [10, we get a similar $Q$ value for $\lambda_{c}=0.1 \mathrm{Mpc} / h$ and $h=0.65$.

\section{PHASE-SPACE DENSITY CONSTRAINTS}

In warm dark matter models like those due to massive neutrinos, an important constraint for late time cosmology results from the finite phase space density [18, 27]. 
For a warm dark matter fermionic particle which decouples when relativistic, the phase space density frozen in is a Fermi-Dirac distribution. The maximum allowed phase space density for a Fermi-Dirac distribution is given by $h^{-3} / 2$. The resulting gravitational distortions of the phase-space sheets can never exceed this phase space limit for collisionless particles. This is a direct consequence of Louiville's theorem as applied to collision-less systems (also called the Vlasov equation).

For the decaying dark matter particle at hand, the maximum phase space density, though finite, can be very large because of the $1 / q$ term. We note that $q$ tends to a constant when the parent particle is relativistic.

The maximum phase space density argument is not the strongest statement that can be made regarding the evolution of collision-less systems. Consider a phase space distribution that is Fermi-Dirac plus a delta-function at some small $q$ value. The maximum phase space density argument would have nothing to say in this case. However, if the total number of particles within the deltafunction spike is small, then we don't expect them to affect the evolution of the collision-less system.

Lynden-Bell 28] showed that collision-less systems have an infinity of conserved quantities. He labeled these $M(>f)$, the mass of particles (or phase space cells) with densities greater than a value $f$. There is however a problem in using $M(>f)$ to make statements about the evolution of a collision-less system. Lets consider the case of a uniform density of particles collapsing to form a galaxy. The observational information we have about the galaxy is never about the fine-grained distribution of the system. We only measure averaged quantities - the coarse-grained distribution. $M(>f)$ is not conserved for coarse-grained distributions; it could increase or decrease. Tremaine et al. [29] proved a theorem using the Boltzmann H-functionals that coarse-graining decreases these $\mathrm{H}$-functionals (concave functions of the distribution function, like the entropy). What about comparing two coarse-grained systems?

Dehnen [30] (see also the early work by Mathur [31]) recently proved that there exists a function - the excess mass function - that always decreases as a result of coarse-graining. The more coarse-grained, the smaller this excess mass function. The excess mass function is very close in spirit to Lynden-Bell's $M(>f)$. It is defined as:

$$
D(f) \equiv \int d^{3} x d^{3} q(F(\mathbf{x}, \mathbf{q})-f) \Theta(F(\mathbf{x}, \mathbf{q})-f)
$$

Let us look at the two important features of this function in a little more detail. First, for collisionless systems $D(f)$ is an invariant. In a collisionless system, the phase space density is conserved along a world-line (solution of the equations of motion). Thus the total "mass" in elements with $F>f$ must be the same (even though the global distribution of this mass could have changed). Second, for collisionless systems, $D(f)$ decreases as a result of coarse-graining. In order to see why this is so, let us coarse-grain by dividing up our phase space into "macro" phase space cells 28]. Each macro-cell of phase space is made up of many (at least more than one) microcells. Suppose that the macro-cell created in this way has $F>f$. Now those micro-cells in this coarse-graining that had densities larger than $f$ don't change $D(f)$. However, the micro-cells with densities smaller than $f$ that get included in $D(f)$ (post coarse-graining) do change $D(f)$. In fact, it can only decrease $D(f)$ because the $F-f$ is negative for them. We can similarly argue that a macro-cell created with $F<f$ also decreases $D(f)$. Thus coarsegraining can only decrease $D(f)$.

This provides a natural way to constrain the inner cores of dark matter halos. $D(f)$ for the halo must be smaller than the $D(f)$ calculated for the primordial distribution function for all values of $f$. We note that this constraint is stronger than demanding that the entropy of the halo be larger than the entropy of the collection of particles that make up the galaxy halo in the early universe. This may be ascertained once the change in entropy is written as $-k \int_{0}^{\infty} d f \Delta D(f) / f[30]$ where $\Delta D(f)$ is the change in the excess mass function.

\section{DARK MATTER HALOS AND THEIR CORES}

As an application of the above discussion consider a halo of DDM particles that has a King profile. These profiles have a core of almost constant density, a roughly $1 / r^{2}$ density run and finally a sharp drop in density to zero. The benefit of these profiles over the usual isothermal profiles is that the King profiles have finite mass.

The small galaxies in the local group have large $Q$ values. From a compilation of Local group dSph galaxies by Mateo [32], we infer that the largest value of $Q$ is observed in Sculptor with a core radius of $110 \mathrm{pc}$ and $1-\mathrm{D}$ stellar velocity dispersion of $6.6 \mathrm{~km} / \mathrm{s}$. Dalcanton and Hogan [33] show that one may assign a $Q$ value of $2 \times 10^{-4} M_{\odot} / \mathrm{pc}^{3} /\left(\mathrm{km} \mathrm{s}^{-1}\right)^{3}$ to this galaxy. They also assign $Q$ values to seven other local group galaxies with measured stellar velocity dispersions between 6 and 11 $\mathrm{km} / \mathrm{s}$ and $M_{v}>-14$. The $Q$ values range between $10^{-5}$ and $10^{-4} M_{\odot} / \mathrm{pc}^{3} /\left(\mathrm{km} \mathrm{s}^{-1}\right)^{3}$.

We choose a King profile for the dark matter halo with a concentration equal to 24 and central density set by the observed stellar profile core and stellar velocity dispersion [34]. The total mass of the halo thus chosen is $2 \times 10^{7} M_{\odot}$. We plot the excess mass function, normalized to the total mass, for this profile in Figure 11 We also plot the primordial excess mass function that is greater than, but barely so, than the halo excess mass function.

The King density profile drops to zero exponentially. The drop is sharp enough that for some small value of $f$, the King excess mass function will always be larger than the primordial one. Therefore, in the above comparison we only look at $f$ values such that $M(>f)<0.99 M(>$ $0)$. Any realistic profile will have a more benign behavior 
at small $f$; indeed, the excess mass function constraint will impose such behavior.

The above exercise indicates that the decay parameters must be such that $Q_{\text {prim }}>2 \times 10^{-5} M_{\odot} / \mathrm{pc}^{3}\left(\mathrm{~km} \mathrm{~s}^{-1}\right)^{-3}$. We note that if the halo of Sculptor is less massive than we have assumed, then $Q_{\text {prim }}$ will have to be larger. If the halo is more massive, then $Q_{\text {prim }}$ can be lower. In scenarios with a small scale cut-off in the power spectrum, the second possibility is more likely because the cut-off leads to a paucity of small mass halos 35 .

We also note that requiring the excess mass function of the galaxy to be smaller than the fine-grained excess mass function in the early universe is a minimal constraint. The final effect on the galaxy of a small primordial phase space density is likely more complicated because of halo mergers.

An interesting exercise at this point is to compare the constraint above to that obtained by demanding that the entropy of the collection of particles that make up the galaxy can only increase. We define entropy for $\mathrm{N}$ indistinguishable particles as $S=-k N \int d^{3} x d^{3} p f(\mathbf{p}, \mathbf{x})\left[\ln \left(f(\mathbf{p}, \mathbf{x}) h^{3}\right)-1\right]$. For dark matter from decays, this works out to $k N \ln \left[2 \pi e^{2-\gamma / 2} m^{4} Q^{-1} h^{-3}\right]$, which is very close to the entropy for an ideal gas of particles with the same $Q$. A comparison with the King profile entropy for Sculptor galaxy yields the result that in order for the entropy to not decrease we must have $Q_{\text {prim }}>$ $10^{-5} M_{\odot} / \mathrm{pc}^{3} /\left(\mathrm{km} \mathrm{s}^{-1}\right)^{3}$, weaker than the constraint obtained from the excess mass function analysis.

The study by Dalcanton and Hogan 33] found that the lowest mass halo core densities could be interpreted as resulting from a finite primordial phase space density. However, they found no compelling argument to attribute to the core density vs. velocity dispersion they found over a wide dynamic range to the effect of a small primordial phase space density.

The effect of a finite $Q$ goes beyond just smoothing the inner cores of small galaxies. Larger galaxies are a result of the merger of smaller galaxies and hence the cumulative effects on the halo density of larger dark matter halos could be substantial. Zentner and Bullock 36. used semi-analytic arguments and the merger tree formalism to show that a small phase space density can lead to a lowered concentration for dark matter halos. For the fermionic warm dark matter candidates, they calculate $c(M)$ for a given virial speed and $Q$. We find that $c(M) \propto Q^{0.63}$ approximates their results well for the range of $Q$ values of interest. The exact relationship between $c(M)$ and $Q$ is more complicated in the present model as compared to the warm dark model case. This is because the constraint on $Q$ depends on the final phase space profile of the halo. A more detailed study is required on this subject.

Why is this important? The concentration of $\Lambda \mathrm{CDM}$ halos as obtained from fits to low surface brightness galaxies (LSBs) falls on the low side. This might be due to two reasons. One, LSBs are extremely strongly bi-

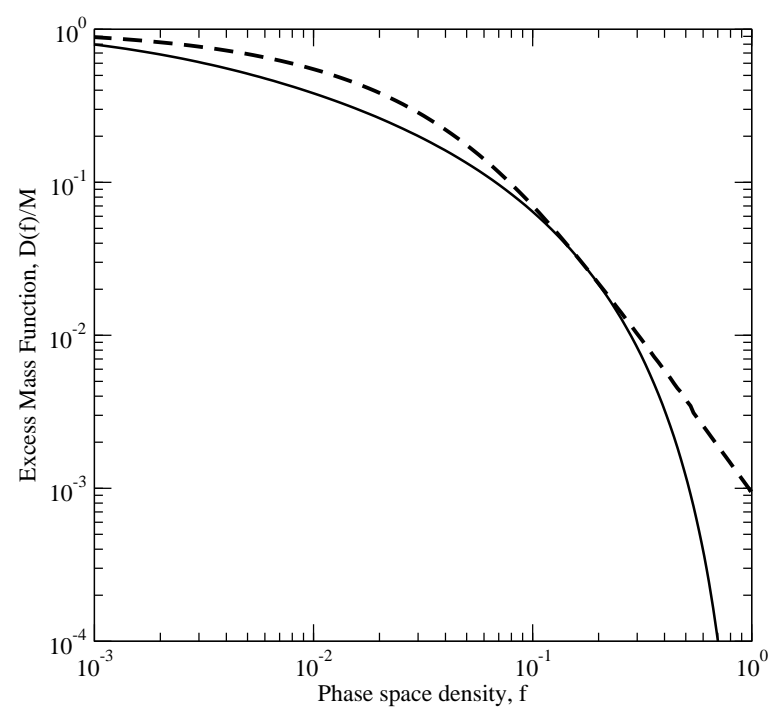

FIG. 1: The solid curve shows the excess mass function for a dark matter halo with a mass of $2 \times 10^{7} M_{\odot}$ and a King profile. The dashed curve shows the excess mass function for the primordial phase space distribution of dark matter from decays with $Q=2 \times 10^{-5} M_{\odot} / \mathrm{pc}^{3}\left(\mathrm{~km} \mathrm{~s}^{-1}\right)^{-3}$.

ased to forming in the lowest concentration halos. Two, CDM is not the correct description of dark matter. As yet, we have no compelling model advocating that the first reason is correct. Clearly, the model presented here has the right features to help explain the observed low concentrations.

The small scale end of the CDM power spectrum has to contend with another issue. CDM predicts a lot of subhalos in the halos of the kind that would host the Milky Way. However, we see almost a factor of ten smaller number of galaxies 37]. On the other hand CDM substructure seems to be required to explain the strong lensing anomalies [38]. Plausible astrophysical solutions 39] to this problem certainly exist, as do more exotic ones. Kamionkowski and Liddle [40] advocated reducing small scale power to explain the lack of small galaxies in the halo of the Milky Way. Zentner and Bullock [36] looked at this issue in detail and worked out the substructure fraction for linear power spectra with a small scale cutoff. Their semi-analytic results applied to our model of dark matter from early decays, and the results of the WDM simulations by Colin et al. 41, suggest that this kind of dark matter will help alleviate the above mentioned discrepancy.

Another problem that has frequently been discussed is the observational case against cuspy halos that CDM simulations predict 42, 43, 44, 45. Low surface brightness and dwarf galaxies seem to be better fit with a density profile that flattens towards the center (e.g., 
[46, 47, 48, 49]). Such evidence for a core in higher mass galaxies is lacking. However, it is also true that that the larger galaxies are dominated by baryons in the centers making it harder to detect a core if it were present. Dark matter from decay of WIMPs can introduce a core in the smallest mass halos $\sim 10^{7} M_{\odot}$ if $Q_{\text {prim }}$ is small enough, $Q_{\text {prim }} \sim 10^{-4} M_{\odot} / \mathrm{pc}^{3} /\left(\mathrm{km} \mathrm{s}^{-1}\right)^{3}$, and reduce the concentration parameter of larger halos. A model with such low $Q$ values would produce a cut-off in the power spectrum given by $\lambda_{c}=0.25 \mathrm{Mpc}$. Is this consistent with having the universe reionize fully at a redshift of six? We explore this in the next section.

\section{REIONIZATION}

Reionization provides a stringent constraint on the small scale power spectrum. The universe is reionized to a redshift of 6 and so there should at least be enough small scale power to do that. The recent WMAP results hint [10] that the optical depth to Thomson scattering might be large $(\sim 0.1)$ indicating early reionzation. If true, this would have dramatic implications for the parameter space of dark matter from decays.

Barkana, Haiman and Ostriker [50] considered the constraints on warm dark matter candidate from cosmological reionization. Their detailed considerations led to the conclusion that WDM with masses larger than about a $\mathrm{keV}$ could reionize the universe at redshift six.

Here we perform a simple analysis to understand the effect of the decay on reionization using the semi-analytic models of Haiman and Holder [51]. The ionized fraction is written as

$$
\begin{gathered}
F_{\mathrm{HII}}(z)=\rho_{\mathrm{b}}(z) \int_{\infty}^{z} d z^{\prime} \epsilon\left[\frac{d F_{\mathrm{coll}, \mathrm{Ib}}}{d z}\left(z^{\prime}\right)\right. \\
\left.+\quad\left(1-F_{\mathrm{HII}}\left(z^{\prime}\right)\right) \frac{d F_{\mathrm{coll}, \mathrm{Ib}}}{d z}\left(z^{\prime}\right)\right] \tilde{V}_{\mathrm{HII}}\left(z^{\prime}, z\right),
\end{gathered}
$$

where $\rho_{\mathrm{b}}$ is the average baryon density and $\tilde{V}_{\mathrm{HII}}\left(z^{\prime}, z\right) \epsilon M$ is the volume of region ionized at redshift $z^{\prime}$ in a halo of mass $M$. The subscripts Ia and Ib 51] refer to different virial temperature ranges. Type Ia halos have temperatures between $10^{4} \mathrm{~K}$ and $2 \times 10^{5} \mathrm{~K}$, while $\mathrm{Ib}$ halos have temperatures higher than $2 \times 10^{5} \mathrm{~K}$. In principle there is also a contribution from halos with virial temperatures smaller than $10^{4} \mathrm{~K}$. This aspect has gotten a lot of attention recently because it could be very important for an early reionization epoch. We are neglecting this contribution here to make a better comparison between CDM and DM from decays. In models with suppressed small scale power, this contribution is small.

The reionization model as written above has two free parameters: $C_{\mathrm{HII}}, \epsilon . C_{\mathrm{HII}}$ is the clumping factor for the IGM predicted by CDM simulations to be of order 10 at high redshifts. In the decaying DM scenario, the clumping factor may be smaller, thus impeding recombinations. For our simple analysis, we will take $C_{\mathrm{HII}}=15$ for CDM and $C_{\mathrm{HII}}=10$ for the DM from decays. A robust calculation will require input from n-body simulations.

The efficiency parameter is the product of the average (over IMF) number of ionizing photons over the lifetime of the source, the escape fraction of ionizing photons and the fraction of baryons converted to sources (stars). We take the efficiency to be 100. Given standard IMFs for metal-rich stars, we expect 4000 ionizing photons per baryon. Thus an efficiency of 100 implies escape fraction times fraction of baryons in stars of $2.5 \%$ in keeping with what is observed in the local universe.

With the above inputs, the $\Lambda \mathrm{CDM}$ model gives a optical depth of 0.091 . For $\lambda_{c}=0.1 \mathrm{Mpc} / h$, we get an optical depth of 0.061 , while for $\lambda_{c}=0.3 \mathrm{Mpc} / h$ we get 0.035 . However, for $\lambda_{c}=1 \mathrm{Mpc} / h$ we get an optical depth of 0.017 , inconsistent with an ionized $z<6$ universe.

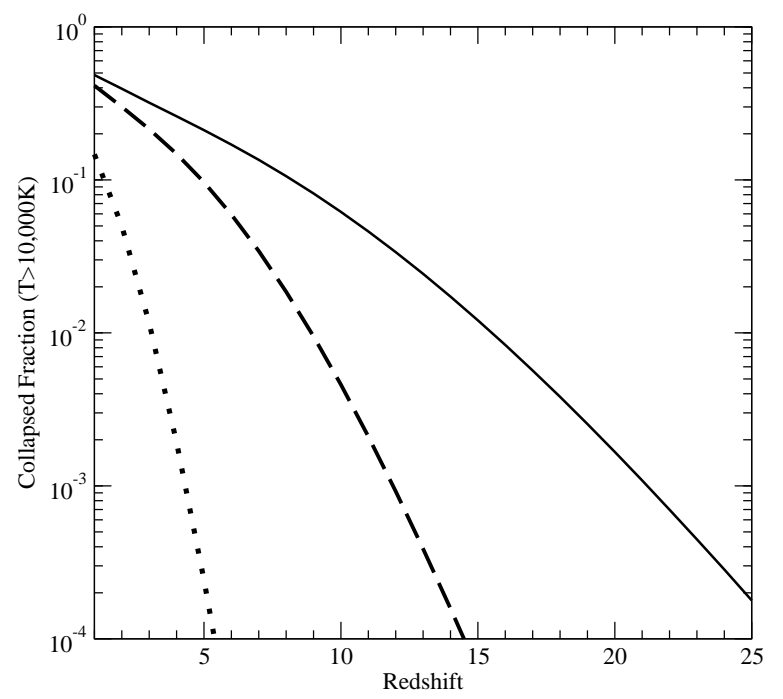

FIG. 2: The curves show the collapsed fractions of halos with virial temperatures larger than $10^{4} \mathrm{~K}$. Below this temperature, the gas can only cool by molecular hydrogen. The solid curve corresponds to a standard $\Lambda$ CDM model with no tilt. The dashed curve corresponds to the same model but with a linear power spectrum modified according to Equation [10] with $\lambda_{c}=0.1 \mathrm{Mpc} / h$. The dotted curve has $\lambda_{c}=1 \mathrm{Mpc} / h$.

The calculations of this section show that in order to be able to reionize the universe by redshift six, we expect that $\lambda_{c} \lesssim 0.3 \mathrm{Mpc} / h$, which implies $Q_{\text {prim }} \gtrsim$ $10^{-5} M_{\odot} / \mathrm{pc}^{3} /\left(\mathrm{km} \mathrm{s}^{-1}\right)^{3}$. The constraint is similar to the one we found in the last section by considering the phase space constraint from local group galaxies. If the universe is reionized at higher redshifts than six, this constraint will tighten considerably.

How reliable are the above calculations? Along with the simplistic modeling of the reionization process, one of the weak points of the above calculation is the calculation 
of the collapsed fraction. We calculate $\sigma^{2}(M)$ using the modified linear power spectrum and use the extended Press-Schechter 52 scheme of Sheth and Tormen [53] to calculate the mass function of dark matter halos. The results of this calculation for the collapsed fraction of halos with $T>10^{4} \mathrm{~K}$ are shown in Figure2 It is unclear that such a calculation will yield accurate results on small scales where the suppression in power becomes important and where structure formation is not fully hierarchical.

\section{MIXED MODELS}

In scenarios of the kind we have discussed above, we may expect some of the DM to be produced during reheating after inflation. Let us take the case of the gravitino DM. If the reheating temperature is around $10^{10}$ $\mathrm{GeV}$ then we expect comparable contribution to the total number density of gravitinos today from the decay and the reheating phase (see Roszkowski and Austri [6] for recent work on early universe constraints on these models). The gravitinos from the reheating phase would behave as Cold Dark Matter particles.

The main impact of these "mixed models" is to soften the small scale effects. This was considered in detail by Profumo et al. [8] for the charged particle decay scenario. We write the dark matter density today as $\rho_{\mathrm{DM}}=f \rho_{\mathrm{DEC}}+(1-f) \rho_{\mathrm{CDM}}$ where $f$ is the fraction of the dark matter that results from the decay. The $z=0$ transfer function for the total dark matter is shown in Figure 3. Note that the suppression on small scales is reduced as expected. The form of the transfer function cannot be trivially obtained from the transfer function for the $f=0$ and $f=1$ cases.

\section{DISCUSSION}

The phenomenology of DDM models is rich and much work remains to be done. To make robust contact with data on small scales we need large scale structure simulations and semi-analytic models. A few detailed simulations in this regard have been run for models with $\mathrm{keV}$ WDM particle [25, 41], with linear power spectrum cutoff on small scales [54], and to understand the dependence of the power spectrum on substructure [55].

We looked at some beneficial features of these models in the previous sections and also the constraints on them. What are the other constraints? Narayanan et al. 56. show that in the WDM scenario, masses smaller than $750 \mathrm{eV}$ are disfavored by the Ly- $\alpha$ data. Recent work by Viel et al. 57 peg the lower limit on the WDM mass at $550 \mathrm{eV}$ at $2 \sigma$. If we naively compare the variance $\sigma(M)$ in the WDM and the present model, then this may be turned into a constraint on the phase space density parameter (see Equation 9), $Q>10^{-4} M_{\odot} / \mathrm{pc}^{3} /\left(\mathrm{km} \mathrm{s}^{-1}\right)^{3}$ corresponding to WDM masses larger than $750 \mathrm{eV}$, and

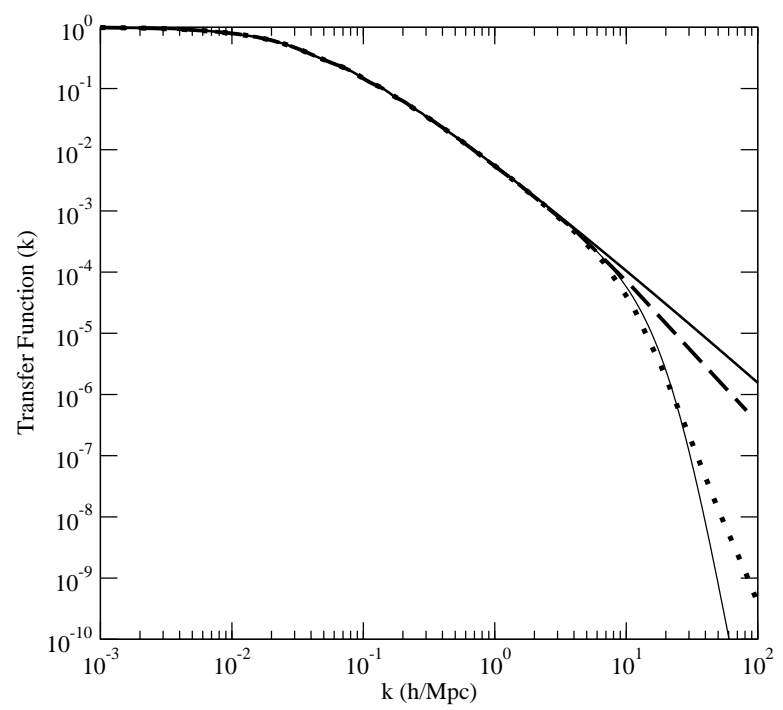

FIG. 3: The curves show the power spectrum for different values of $f$, the fraction of dark matter today that came from decays. The solid curve shows the $f=0$ case (CDM). The dashed curve shows the $f=0.5$ case while the dotted curve shows the $f=1$ case. It is clear that the suppression on small scales is much reduced for the $f=0.5$ case. For comparison, we also plot (see thin solid curve) the transfer function for a $1 \mathrm{keV}$ Warm Dark Matter model.

$Q>5 \times 10^{-5} M_{\odot} / \mathrm{pc}^{3} /\left(\mathrm{km} \mathrm{s}^{-1}\right)^{3}$ corresponding to WDM masses larger than $550 \mathrm{eV}$.

Thus, we may summarize the present constraints on models where the bulk of dark matter today results from decays with a lifetime of about a year or smaller as $Q \gtrsim 10^{-4} M_{\odot} / \mathrm{pc}^{3} /\left(\mathrm{km} \mathrm{s}^{-1}\right)^{3}$. Future comparisons with data will require large simulations to understand structure formation in these models; it does not proceed in a bottom-up hierarchical manner 25, 41, 54].

Can we push down to $100 \mathrm{kpc}$ or even $10 \mathrm{kpc}$ cut-off scales? Strong lensing flux anomaly technique is a sensitive probe of the amount of substructure 13]. Robust limits from this technique will require detailed theoretical understanding of issues such as the anisotropic distribution of substructure in dark matter halos [58]. Weak lensing constraints from future surveys is another promising avenue to learn more about dark matter on these small scales [14].

\section{CONCLUSIONS}

In this paper, we have explored the important cosmological consequences of dark matter from early decays the cut-off in the power spectrum on small scales, and the limit on the phase space density of dark matter in halos. 
The phenomenology is rich and there are multiple ways to search for the effect of such dark matter on structure formation. We pointed out that these models can suppress small scale substructure, create constant density cores in small mass halos due to the phase space constraint and reduce the concentration of larger mass halos. The models that give rise to these early decays in supersymmetric theories are natural and they inherit the correct cosmological abundance from the WIMPs that decay into them. Future observations of structure on small scales may be able to distinguish between cold dark matter and the dark matter from early decays.

\section{ACKNOWLEDGMENTS}

I thank Jose Cembranos, Jonathan Feng, Fumihiro Takayama and Andrew Zentner for discussions. I thank James Bullock for many discussions about the problems on small scales, Neal Dalal for discussions during the early phase of this project and Arvind Rajaraman for convincing me about the usefulness of the excess mass function. We acknowledge the use of CMBfast code [22] for this work.
[1] J. L. Feng, A. Rajaraman, and F. Takayama, Phys. Rev. Lett. 91, 011302 (2003), hep-ph/0302215.

[2] J. L. Feng, A. Rajaraman, and F. Takayama, Phys. Rev. D68, 085018 (2003), hep-ph/0307375.

[3] J. L. Feng, A. Rajaraman, and F. Takayama, Phys. Rev. D68, 063504 (2003), hep-ph/0306024.

[4] J. L. Feng, S.-f. Su, and F. Takayama, Phys. Rev. D70, 063514 (2004), hep-ph/0404198.

[5] J. L. Feng, S. Su, and F. Takayama, Phys. Rev. D70, 075019 (2004), hep-ph/0404231.

[6] L. Roszkowski and R. Ruiz de Austri (2004), hep$\mathrm{ph} / 0408227$.

[7] R. Lamon and R. Durrer (2005), hep-ph/0506229.

[8] S. Profumo, K. Sigurdson, P. Ullio, and M. Kamionkowski, Phys. Rev. D71, 023518 (2005), astro-ph/0410714.

[9] K. Sigurdson and M. Kamionkowski, Phys. Rev. Lett. 92, 171302 (2004), astro-ph/0311486.

[10] A. Kogut et al. (2003), astro-ph/0302213.

[11] J. A. R. Cembranos, J. L. Feng, A. Rajaraman, and F. Takayama (2005), hep-ph/0507150.

[12] A. Mesinger, R. Perna, and Z. Haiman, Astrophys. J. 623, 1 (2005), astro-ph/0501233.

[13] N. Dalal and C. S. Kochanek (2002), astro-ph/0202290.

[14] D. Dolney, B. Jain, and M. Takada, Mon. Not. Roy. Astron. Soc. 352, 1019 (2004), astro-ph/0401089.

[15] W. B. Lin, D. H. Huang, X. Zhang, and R. H. Brandenberger, Phys. Rev. Lett. 86, 954 (2001), astro$\mathrm{ph} / 0009003$.

[16] J. Hisano, K. Kohri, and M. M. Nojiri, Phys. Lett. B505, 169 (2001), hep-ph/0011216.

[17] R. Kitano and I. Low (2005), hep-ph/0503112.

[18] C. J. Hogan and J. J. Dalcanton, Phys. Rev. D62, 063511 (2000), astro-ph/0002330.

[19] M. Kawasaki, G. Steigman, and H.-S. Kang, Nucl. Phys. B403, 671 (1993).

[20] H.-S. Kang, M. Kawasaki, and G. Steigman, Nucl. Phys. B402, 323 (1993).

[21] C.-P. Ma and E. Bertschinger, Astrophys. J. 455, 7 (1995), astro-ph/9506072.

[22] U. Seljak and M. Zaldarriaga, Astrophys. J. 469, 437 (1996), astro-ph/9603033.

[23] J. R. Bond, G. Efstathiou, and J. Silk, Phys. Rev. Lett. 45, 1980 (1980).

[24] R. H. Brandenberger, N. Kaiser, and N. Turok, Phys. Rev. D36, 2242 (1987).
[25] P. Bode, J. P. Ostriker, and N. Turok, Astrophys. J. 556, 93 (2001), astro-ph/0010389.

[26] C.-P. Ma, Astrophys. J. 471, 13 (1996), astroph/9605198.

[27] S. Tremaine and J. E. Gunn, Phys. Rev. Lett. 42, 407 (1979).

[28] D. Lynden-Bell, Memoires of the Societe Royale des Sciences de Liege 55, 143 (1967).

[29] S. Tremaine, M. Henon, and D. Lynden-Bell, Mon.Not.Roy.As.Soc. 219, 285 (1986).

[30] W. Dehnen, Mon. Not. Roy. Astron. Soc. 360, 869 (2005), astro-ph/0504246.

[31] S. D. Mathur, Mon.Not.Roy.As.Soc. 231, 367 (1988).

[32] M. L. Mateo, Annu. Rev. Astron. Astrophys. 36, 435 (1998).

[33] J. J. Dalcanton and C. J. Hogan, Astrophys. J. 561, 35 (2001), astro-ph/0004381.

[34] C. Pryor and J. Kormendy, Astron. J. 100, 127 (1990).

[35] J. S. Bullock and A. R. Zentner, Bulletin of the American Astronomical Society 34, 1170 (2002).

[36] A. R. Zentner and J. S. Bullock, Astrophys. J. 598, 49 (2003).

[37] B. Moore et al., Astrophys. J. 524, L19 (1999).

[38] C. S. Kochanek and N. Dalal, Astrophys. J. 610, 69 (2004), astro-ph/0302036.

[39] J. S. Bullock, A. V. Kravtsov, and D. H. Weinberg, Astrophys. J. 539, 517 (2000).

[40] M. Kamionkowski and A. R. Liddle, Phys. Rev. Lett. 84, 4525 (2000), astro-ph/9911103.

[41] P. Colín, V. Avila-Reese, and O. Valenzuela, Astrophys. J. 542, 622 (2000).

[42] B. Moore, Nature (London) 370, 629 (1994).

[43] R. A. Flores and J. R. Primack, Astrophys. J. Lett. 427, L1 (1994).

[44] A. V. Kravtsov, A. A. Klypin, J. S. Bullock, and J. R. Primack, Astrophys. J. 502, 48 (1998), astro$\mathrm{ph} / 9708176$.

[45] B. Moore, F. Governato, T. Quinn, J. Stadel, and G. Lake, Astrophys. J. Lett. 499, L5+ (1998).

[46] D. T. F. Weldrake, W. J. G. de Blok, and F. Walter, Mon.Not.Roy.As.Soc. 340, 12 (2003).

[47] R. A. Swaters, M. A. W. Verheijen, M. A. Bershady, and D. R. Andersen, Astrophys. J. 587, L19 (2003), astroph/0303052.

[48] J. D. Simon, A. D. Bolatto, A. Leroy, L. Blitz, and E. L. Gates, Astrophys. J. 621, 757 (2005), astro-ph/0412035. 
[49] K. Spekkens, R. Giovanelli, and M. P. Haynes, Astron. J. 129, 2119 (2005).

[50] R. Barkana, Z. Haiman, and J. P. Ostriker (2001), astro$\mathrm{ph} / 0102304$.

[51] Z. Haiman and G. P. Holder, Astrophys. J. 595, 1 (2003), astro-ph/0302403.

[52] W. H. Press and P. Schechter, Astrophys. J. 187, 425 (1974).

[53] R. K. Sheth and G. Tormen, Mon. Not. Roy. Astron. Soc. 329, 61 (2002), astro-ph/0105113.

[54] M. White and R. A. C. Croft, Astrophys. J. 539, 497
(2000).

[55] V. R. Eke, J. F. Navarro, and M. Steinmetz, Astrophys. J. 554, 114 (2001).

[56] V. K. Narayanan, D. N. Spergel, R. Davé, and C. Ma, Astrophys. J. Lett. 543, L103 (2000).

[57] M. Viel, J. Lesgourgues, M. G. Haehnelt, S. Matarrese, and A. Riotto, Phys. Rev. D71, 063534 (2005), astro$\mathrm{ph} / 0501562$.

[58] A. R. Zentner, A. V. Kravtsov, O. Y. Gnedin, and A. A. Klypin (2005), astro-ph/0502496. 